\title{
Clinical evaluation of RB1 genetic testing reveals novel mutations in Vietnamese patients with retinoblastoma
}

\author{
CHINH QUOC HOANG ${ }^{1-3 *}$, HONG-QUAN DUONG ${ }^{2,4^{*}}$, NGUYEN THANH NGUYEN ${ }^{5}$, \\ SY ANH HAO NGUYEN ${ }^{6}$, CUONG NGUYEN ${ }^{1,7}$, BO DUY NGUYEN ${ }^{8}$, LAN TUYET PHUNG $^{8}$, \\ DUNG THUY NGUYEN ${ }^{8}$, CHAU THI MINH PHAM ${ }^{9}$, TRANG LE DOAN ${ }^{9}$ and MAI HOANG TRAN ${ }^{2,5}$ \\ ${ }^{1}$ Vinmec Hi-Tech Center, Vinmec Healthcare System; ${ }^{2}$ Department of Cancer Research, Vinmec Research Institute of \\ Stem Cell and Gene Technology; ${ }^{3}$ Center for Experimental Biology, National Center for Technological Progress; \\ ${ }^{4}$ Laboratory Center, Hanoi University of Public Health; ${ }^{5}$ Department of Translational Biomedical Informatics, \\ Vingroup Big Data Institute; ${ }^{6}$ Institute for Preventive Medicine and Public Health, Hanoi Medical University; \\ ${ }^{7}$ LOBI Vietnam Ltd.; ${ }^{8}$ Department of Pediatrics 3, Vinmec International Hospital in Times City, Vinmec Healthcare System; \\ ${ }^{9}$ Department of Pediatric Ophthalmology, Vietnam National Eye Hospital, Hanoi 100000, Vietnam
}

Received January 21, 2021; Accepted June 8, 2021

DOI: $10.3892 / \mathrm{mco} .2021 .2344$

\begin{abstract}
Clinical evaluation of the genetic testing strategy is essential for ensuring the correct determination of mutation carriers. The current study retrospectively analyzed genetic and clinicopathological data from 62 Vietnamese patients with retinoblastoma $(\mathrm{RB})$ referred to the Vinmec Hi-Tech Center for RB transcriptional corepressor $1(R B 1)$ genetic testing between 2017 and 2019. The present study aimed to evaluate the sensitivity of the Next Generation Sequencing (NGS) method to identify novel $R B 1$ mutations, and to consider using age at diagnosis as a risk factor. Genomic DNA was analyzed with custom panel based targeted NGS. NGS was performed on the Beijing Genomics Institute (BGI) sequencing platform, and pathogenic or likely pathogenic variants were confirmed by Sanger sequencing, quantitative PCR (qPCR) or Multiplex Ligation-dependent Probe Amplification assay (MLPA). Constitutional $R B 1$ variants were identified in $100 \%(25 / 25)$ of the bilateral cases, while several common previously reported $R B 1$ mutations were also recorded. In addition, in Vietnamese patients with $\mathrm{RB}$, nine novel $R B I$ mutations were identified. Children aged between 0-36 months
\end{abstract}

Correspondence to: Dr Chinh Quoc Hoang, Vinmec Hi-Tech Center, Vinmec Healthcare System, 458 Minh Khai, Hai Ba Trung, Hanoi 100000, Vietnam

E-mail: chinhhq83@gmail.com

Dr Hong-Quan Duong, Laboratory Center, Hanoi University of Public Health, 1A Duc Thang, North Tu Liem, Hanoi 100000, Vietnam

E-mail: dhq@huph.edu.vn

*Contributed equally

Key words: retinoblastoma, RB transcriptional corepressor 1, next generation sequencing, sensitivity, novel mutations, risk-stratified factors were more likely to be $R B 1$ carriers compared with those aged $>36$ months. The current findings indicated that the NGS method implemented in the Vinmec Hi-Tech Center was highly accurate, and age at diagnosis may be used to assess the risk of hereditary RB. Furthermore, the newly identified $R B 1$ mutations may provide additional data to improve the current understanding of the mechanisms underlying $R B 1$ inactivation and the development of rapid assays for detecting $R B 1$ mutations. Overall, the present study suggested that NGS may be applied for detecting germline $R B 1$ mutations in routine clinical practice.

\section{Introduction}

Retinoblastoma (RB) is the most common intraocular malignancy, characterized by high mortality if not detected early and treated promptly. Early diagnosis and intervention play a key role in the successful treatment of RB (1). Delayed diagnosis of $\mathrm{RB}$ for $>6$ months from the first clinical sign has been reported to be associated with a mortality rate of $\sim 70 \%$ (2). Therefore, although in patients with RB diagnosed at stage $\mathrm{A}$ of the disease the eyes or vision can be salvaged, currently, there is not an effective treatment approach for those diagnosed at stage $\mathrm{E}(3,4)$.

$\mathrm{RB}$ is considered as a monogenetic hereditary type of cancer since $97 \%$ of RB cases are caused by the disruption of the RB transcriptional corepressor $1(R B 1)$ tumor suppressor gene (5). The $R B 1$-encoded protein (pRB) acts as a scaffold protein, which interacts with other proteins to regulate multiple cellular processes essential for cell fate and function. Consequently, $R B 1$ deficiency may predispose cells to tumorigenesis. In fact, it has been reported that $R B 1$ inactivation is detected in several types of cancer. Since pRB interacts with other proteins through cyclin folds in the $\mathrm{N}$-terminus and pocket domain, and intrinsically disordered structures in the C-terminus, a wide spectrum of mutations dispersed throughout the RB1 gene has been identified in patients with cancer (6). 
It has been also reported that $45 \%$ of patients with $\mathrm{RB}$ are suffering from the inherited type of the disease ( $R B 1$ carriers), where the first allele of $R B 1$ is mutated during preconception or shortly after conception, predisposing the child to retinal tumorigenesis (7). The remaining $R B 1$ allele is functionally lost during retinal development, promoting the initiation of RB in either both eyes (bilateral RB) or one eye (unilateral RB) with multifocal tumors. 'Non-heritable or sporadic RB' (non-RBI carriers) is commonly referred to as patients without germline RB1 mutations, who usually present with unifocal tumors in one eye (8). Patients with hereditary RB exhibit a worse prognosis, are sensitive to certain treatments, have a high risk of developing second primary malignancies and can pass the mutations on to their offspring $(3,9)$. Therefore, identifying germline $R B 1$ mutations is of great importance for implementing the appropriate treatment approach, and assessing the risk of developing second primary malignancies, both secondary RB and other primary malignancies, in patients with $\mathrm{RB}$, and the risk of $\mathrm{RB}$ onset in the patient's relatives.

Genetic testing and counseling (GTC) are recommended for all patients with $\mathrm{RB}$ and are integrated in the management of $\mathrm{RB}$ in developed countries (10), thus resulting in high survival rate of patients with $\mathrm{RB}$ and cost-effective medical treatments for RB $(11,12)$. GTC for patients with bilateral or familial RB is straightforward. However, for unilateral, non-familial RB cases, GTC is not as easy due to the low risk of $R B l$ mutation carriers, and the inefficient detection of germline $R B 1$ mosaicism (13). Genetic testing for $R B l$ is a time-consuming and expensive procedure given the large size of the $R B 1$ gene, which can be inactivated by multiple mutations, and the absence of mutational hotspots $(14,15)$. Direct sequencing is widely applied for detecting $R B 1$ mutations, however, this method is not recommended for identifying low allelic-fraction variants (16). For the detection of these types of variants, PCR can be applied only when the mutations are already known. In addition, Multiplex Ligation-dependent Probe Amplification (MLPA), quantitative PCR (qPCR) or array Comparative Genomic Hybridization (aCGH) can identify large $R B 1$ rearrangements. The combination of the aforementioned methods is essential for detecting all possible RBI mutations (17-19). Recently, Next Generation Sequencing (NGS) has been implemented as rapid and effective strategy for identification of $R B 1$ mutations since all variations can be detected in a single test, thus providing a number of advantages, including high sensitivity and cost-effectiveness (20-25). In Vietnam, Sanger sequencing coupled with MLPA (SS-MLPA) could detect germline RB1 mutations in $82-84 \%$ of bilateral cases $(26,27)$. Nevertheless, the sensitivity of NGS in a routine clinical practice remains unknown.

The two-hit hypothesis suggests that patients with bilateral or unilateral multifocal RB, and/or diagnosed at an early age are more likely to carry germline $R B 1$ mutations (28). This hypothesis is supported by clinical data. Therefore, a study demonstrated that up to $100 \%$ of patients with bilateral carried germline $R B 1$ mutations, and their age at diagnosis was 10 months younger compared with that of the unilateral cases (13). Bilateral and unilateral eye diseases account for 40 and $60 \%$ of all RB cases (7), respectively. All bilateral RB cases are considered heritable, whereas $\sim 15 \%$ of unilateral cases carry constitutional $R B 1$ mutations $(29,30)$. Since age at diagnosis is younger in bilateral cases, previous studies have associated age at diagnosis with germline $R B 1$ status in order to predict age associated with increased risk of patients with unilateral disease being $R B 1$ carriers. Unfortunately, these studies yielded conflicting results (31-34).

The current study retrospectively analyzed the clinicopathological and genetic data of patients with RB to evaluate the sensitivity of NGS for detecting constitutional $R B I$ variants, to detect novel germline $R B 1$ mutations, and to consider age at diagnosis as a risk factor for patients being $R B 1$ carriers.

\section{Materials and methods}

Patients and samples. In the current retrospective study, a total of 62 patients with RB were included, who were referred to Department of Cancer Research, Vinmec Research Institute of Stem Cell and Gene Technology and the Department of Medical Genetics, Vinmec Hi-Tech Center for genetic testing between 2017 and 2019. Signed informed consents were obtained from parents/caregivers of all subjects and the study was approved by the Vinmec's Institutional Review Board. All data, including age at diagnosis, sex, tumor stages, laterality and family history were retrieved from the patients' medical records. Genetic testing for germline $R B l$ variants was performed at the Beijing Genomics Institute (BGI), HongKong. The workflow for analyzing germline $R B 1$ variants is illustrated in Fig. 1.

Preparation of tissue samples, NGS and variant calling. Genomic DNA (gDNA) was extracted from peripheral blood mononuclear cells and its concentration was quantified using a Qubit Fluorometer (Thermo Fisher Scientific, Inc.). Subsequently, gDNA was fragmented, indexed and amplified. The $R B 1$ promoter and all its exons, plus 20 nucleotides proximal to either $5^{\prime}$ or $3^{\prime}$ of each exon, were captured by a BGI chip. Library size and quantity were verified using Qubit ${ }^{\circledR} 2.0$ (Thermo Fisher Scientific, Inc.) and an Agilent Bioanalyzer 2100 (Agilent Technologies, Inc.). Sequencing was performed on the BGIseq platform. All samples identified with pathogenic or likely pathogenic variants were confirmed by Sanger Sequencing, qPCR or MLPA.

Bioinformatics analysis. The sequence reads were aligned to the human reference genome (GRCh37/Hg19) using the Burrows-Wheeler Aligner. Single nucleotide variants, insertion/deletion variants (InDel) and copy number variations were identified using the BGI internal NGS software (BGISEQ-500).

Variant annotation. Interpretation of germline variants followed the American College of Medical Genetics and Genomics (ACMG) standards and guidelines (35). The variants were designated according to nomenclature and the recommendations of the Human Genome Variation Society (36). Public databases, including Clinvar (37), Universal Mutation Database (38), Leiden Open Variation Database (LOVD) (39) and ARUP (40) were used for data analysis. The SIFT (41), POLYPHEN2 (42) and Mutation taster (43) tools were applied to predict deleterious mutations, while variants were classified using the Varsome (44) and InterVar (45) classification tools.

Identification of novel variants. All variants identified by the BGI laboratory were annotated with the Ensemble Variant Effect 


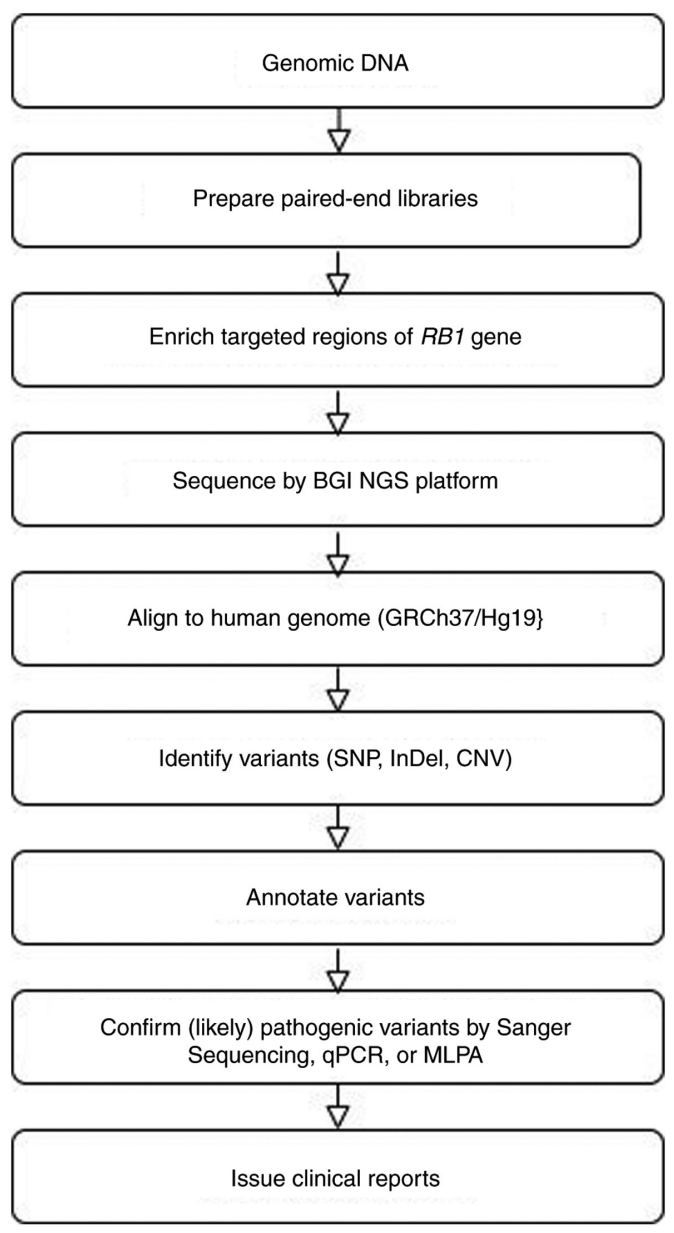

Figure 1. Workflow for analyzing $R B 1$ germline variants. RB1, RB transcriptional corepressor 1; NGS, next generation sequencing; NSP, single nucleotide polymorphism; InDel, insertion/deletion; CNV, copy number variant; qPCR, quantitative PCR; MLPA, Multiplex Ligation-dependent Probe Amplification; BGI, Beijing Genomics Institute.

Predictor (EVEP) tool (46). Variants with existing ID were checked on corresponding databases. The unmatched variants and those without existing ID were screened on PubMed. Variants not previously reported in public databases and PubMed were considered as novel ones. The allele frequency of the identified novel variants was investigated in four databases, including the 1000 Genomes Project (browser.1000genomes.org), the Exome Sequencing Project (esp.gs.washington.edu/drupal), the Genome Aggregation Database and the Vietnamese Genetic Variation Database (47). Deleterious novel variants were evaluated by Combined Annotation Dependent Depletion (CADD) (48) and EVEP, while their pathogenicity was interpreted using VarSome.

Statistical analysis. The differences between the characteristics of $R B I$ and non- $R B 1$ carriers were compared using a $\chi^{2}$ test, Fisher's exact test or Student's t-test. Fisher's exact test and odds ratio (OR) were calculated using calculators provided by the Social Science Statistics website (49) and MedCalc statistical software (50), respectively.

\section{Results}

Characteristics of RBland non-RB1 carriers. A total of 25 bilateral and 37 unilateral RB cases were included in the present study. There was no statistically significant difference in the total number of $R B 1$ and non- $R B 1$ carriers, as well as in sex distribution between the two groups. However, the mean age at diagnosis of $R B 1$ carriers was significantly younger compared with that of non- $R B 1$ carriers (22.14 vs. 32.66 months). Although $80 \%$ of all patients were diagnosed with stage $\mathrm{E} R \mathrm{RB}$, the distribution of the RB stages was not statistically different between $R B 1$ and non- $R B 1$ carriers. Additionally, the proportion of $R B 1$ carriers was notably higher in patients with bilateral $R B$ and significantly decreased in the unilateral cases. Constitutional RB1 mutations were detected in $100 \%(25 / 25)$ and $27 \%(10 / 37)$ of patients with bilateral and unilateral RB, respectively (Tables I and II), suggesting a sensitivity rate of $100 \%$ for detecting germline $R B I$ variants using the NGS technology.

Novel RB1 germline mutations. A total of 28 distinct variants, including four recurrent and 24 non-recurrent ones, were identified in 56\% (35/62) of patients with. RB. In addition, the four recurrent mutations were found in $33 \%$ (12/35) of $R B 1$ carriers. Point and small InDel mutations in $R B I$ were dispersed along the gene. However, large rearrangements were only identified in or nearby the pocket domain. The majority of mutations (26/28) were located in the $\mathrm{N}$-terminus or pocket domain of pRB (Fig. 2). The EVEP tool predicted that all these variations, except one (c.83C $>\mathrm{G}$ ), exerted a highly disruptive effect in $\mathrm{pRB}$ and were classified as pathogenic variants using the Clinvar, Cosmic or Varsome databases. The c. $83 \mathrm{C}>\mathrm{G}$ mutation was concurrently found with an exon 12 duplication in one patient with bilateral RB. Nonsense and slice mutations were the two most frequent mutations, identified in $34(12 / 35)$ and $31 \%$ (11/35) of all RBI carriers, respectively. Additionally, large rearrangements, frameshift and missense mutations were detected in 17 (6/35), $11.4(4 / 35)$ and $8.6 \%$ (3/35) of $R B I$ carriers, respectively (Tables I and III).

The novel variants were defined by screening $28 R B 1$ mutations into the EVEP tool. Among them, $16 R B 1$ alterations exerted Variation ID on the Clinvar, Cosmic or LOVD databases. For the remaining 12 unidentified variants, screening on the PubMed platform was carried out. The analysis revealed three large rearrangement, including Ex1_27 DEL, Ex24 DEL and Ex13_18 DUP. Therefore, nine variants were considered as novel. These nine variations, including one large rearrangement, one nonsense, four slice and three frameshift mutations, were all null mutations, identified in seven patients with bilateral and two with unilateral RB. These variants were located at either the pocket (5/9) or N-terminus (4/9) domain. Furthermore, the EVEP tool predicted that these variants could have a significant disruptive effect on pRB, and all, except EX12 DUP, were also predicted to be among the top $1 \%$ of the most deleterious variants in the human genome by CADD. Finally, these mutations were not found in polymorphism databases, and were classified as pathogenic by VarSome (Table III; Fig. 2).

Association between age at diagnosis and the genetic status of patients with $R B$. The proportion of $R B 1$ and non- $R B 1$ carriers with an age at diagnosis of 0-36 and $>36$ months is presented in Table IV. It was found that the proportion of $R B 1$ carriers with age at diagnosis of 0-36 months was notably higher compared with those of $>36$ months. Consequently, the relative risk of children aged between 0-36 months being $R B 1$ carriers was significantly higher than that of children $>36$ months 


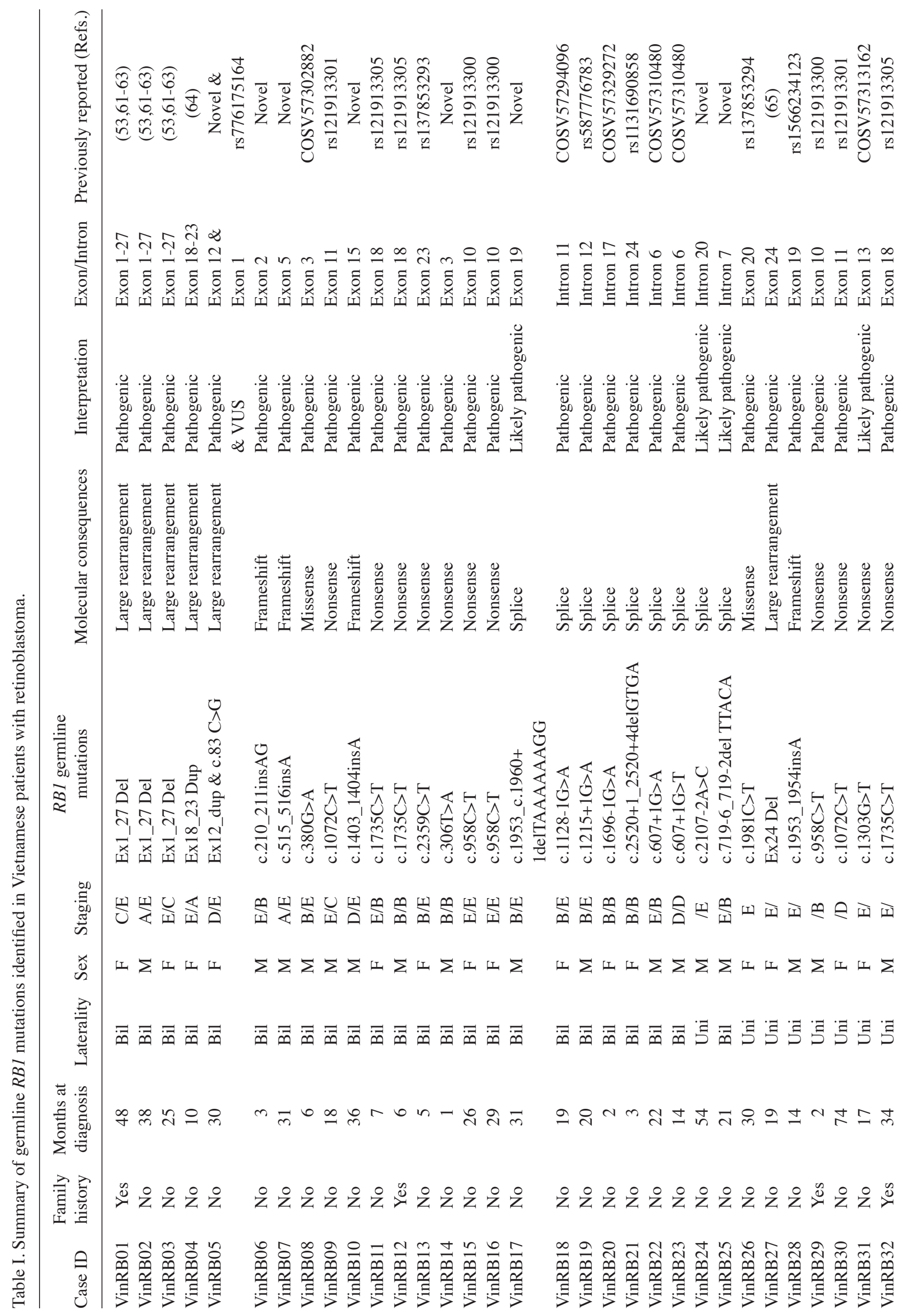




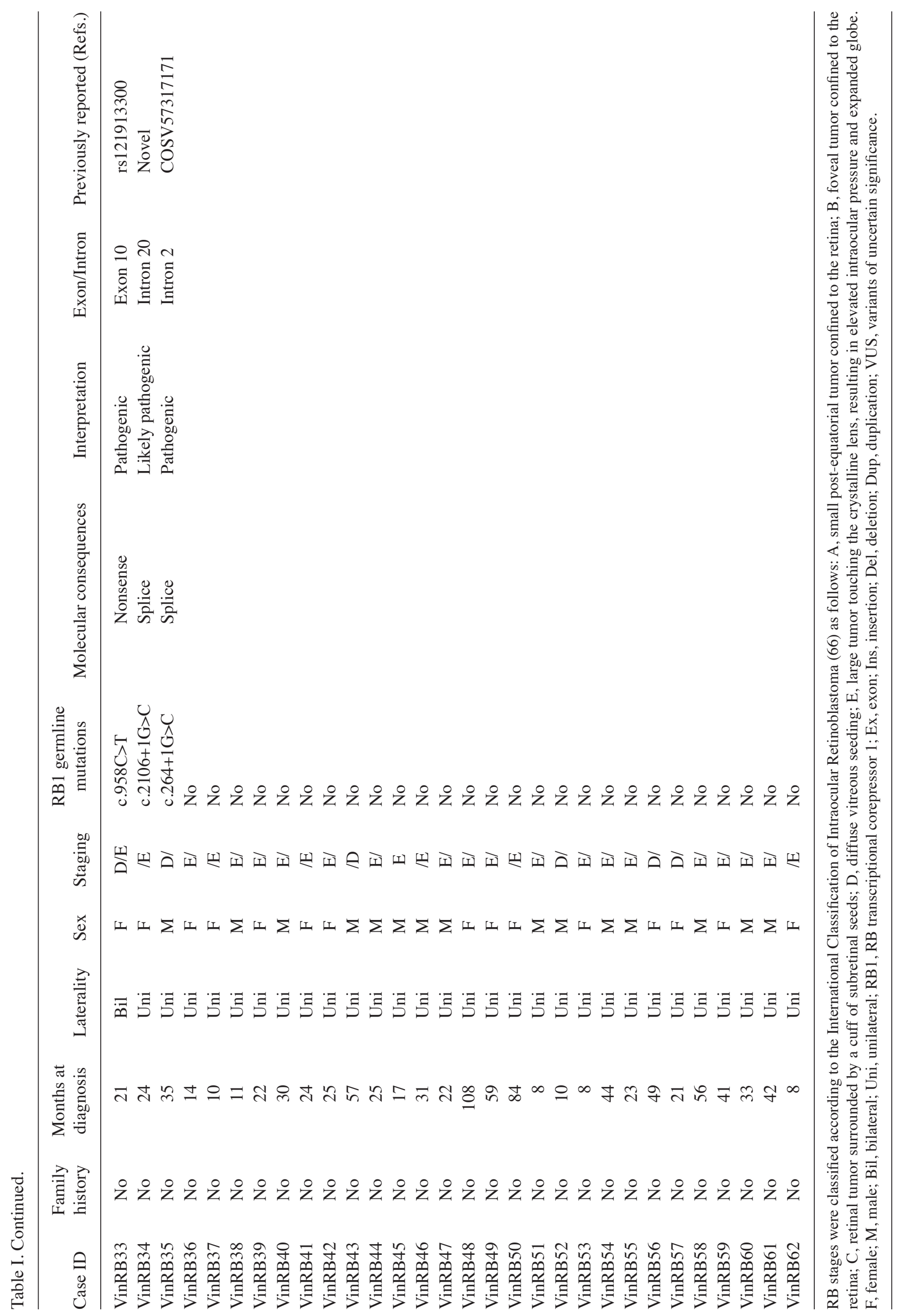


Table II. Clinicopathological distribution of $R B 1(\mathrm{n}=35)$ and non- $R B 1(\mathrm{n}=27)$ carriers by family history, laterality, sex, age at diagnosis and stage of RB tumorigenesis.

\begin{tabular}{|c|c|c|c|c|}
\hline Characteristics & RB1 carriers $(n=35)$ & Non-RB1 carriers $(n=27)$ & Both $(n=62)$ & P-value \\
\hline Family history, n (\%) & $4(100.0)$ & $0(0.0)$ & $4(100.0)$ & \\
\hline Laterality, n (\%) & & & & $0.001^{\mathrm{a}}$ \\
\hline Bilateral RB & $25(100.0)$ & $0(0.0)$ & $25(100.0)$ & \\
\hline Unilateral RB & $10(27.0)$ & $27(73.0)$ & $37(100.0)$ & \\
\hline Sex, n (\%) & & & & $0.87^{\mathrm{b}}$ \\
\hline Female & $17(57.0)$ & $13(43.0)$ & $30(100.0)$ & \\
\hline Male & $18(56.0)$ & $14(44.0)$ & $32(100.0)$ & \\
\hline Mean age, months & 22.14 & 32.67 & & $0.04^{\mathrm{c}}$ \\
\hline RB stage, $\mathrm{n}(\%)$ & & & & $0.80^{\mathrm{a}}$ \\
\hline A & $0(0.0)$ & $0(0.0)$ & $0(0.0)$ & \\
\hline B & $4(11.4)$ & $1(3.7)$ & $5(8.0)$ & \\
\hline $\mathrm{C}$ & $0(0.0)$ & $0(0.0)$ & $0(0.0)$ & \\
\hline $\mathrm{D}$ & $3(8.6)$ & $4(14.8)$ & $7(11.3)$ & \\
\hline E & $28(80.0)$ & $22(81.5)$ & $50(80.6)$ & \\
\hline
\end{tabular}

${ }^{\mathrm{a} C a l c u l a t e d ~ u s i n g ~ F i s h e r ' s ~ e x a c t ~ t e s t . ~}{ }^{\mathrm{b}}$ Calculated using $\chi^{2}$ test. ${ }^{\mathrm{c} C a l c u l a t e d}$ using Student's t-test. RB stages were classified according to the International Classification of Intraocular Retinoblastoma (66) as follows: A, small post-equatorial tumor confined to the retina; B, foveal tumor confined to the retina; C, retinal tumor surrounded by a cuff of subretinal seeds; D, diffuse vitreous seeding; E, large tumor touching the crystalline lens, resulting in elevated intraocular pressure and expanded globe. RB, retinoblastoma; RB1, RB transcriptional corepressor 1 .

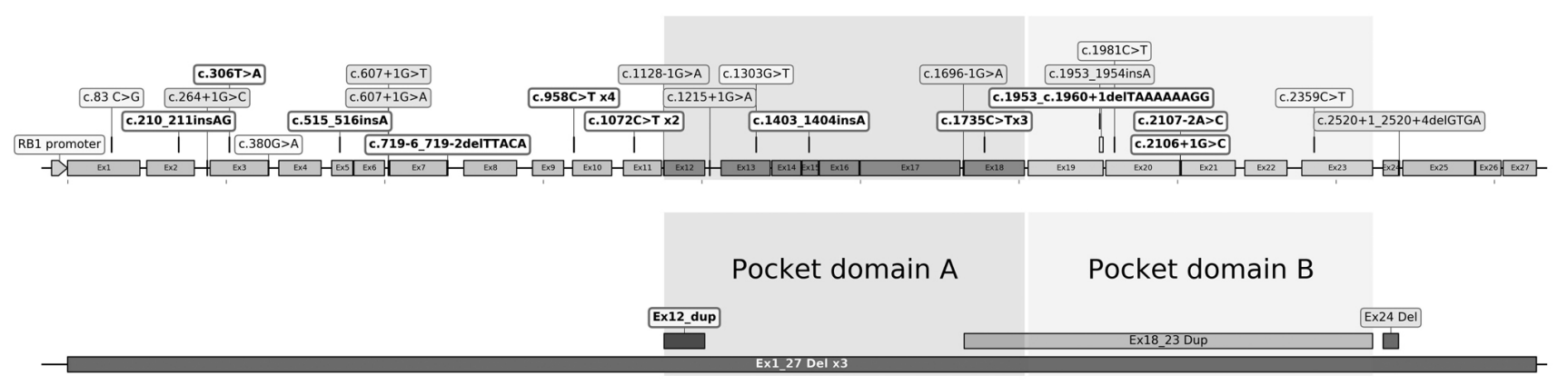

Figure 2. Distribution of 28 distinctly constitutional $R B 1$ variants, including point and InDel alterations (top) and large rearrangements (bottom). Bold variations in white boxes indicate newly identified and recurrent mutations. Recurrent mutations were marked with numbers of carriers (x $n$ ). RB1, RB transcriptional corepressor 1; InDel, insertion/deletion; Ex, exon; Dup, duplication.

(63.3 vs. $36 \%$; OR=3.88; 95\% CI=1.04-14.4; Table IV). In terms of unilateral cases only, although the number of $R B I$ carriers was reduced compared with the non- $R B 1$ ones, the difference between the two groups was not statistically significant. Accordingly, the relative risk of children aged between 0-36 months suffering from the inherited form of the disease was not markedly different compared with children $>36$ months of age (30.8 vs. $18 \%$; OR=2; $95 \% \mathrm{CI}=0.35=11.4$; Table IV).

\section{Discussion}

Integrated $R B 1$ genetic testing for the management of RB may reduce $\mathrm{RB}$-associated mortality and treatment costs. However, the test sensitivity rate should be $\geq 90 \%$ to ensure a negative result would indicate a low risk of hereditary RB. In the current study, patients who were diagnosed with bilateral RB were used as a positive control group to evaluate the sensitivity of NGS in routine clinical practice. The detection rate of NGS was $100 \%$ for patients with bilateral $\mathrm{RB}$, while all common $R B 1$ mutation types were detected. Additionally, nine novel pathogenic mutations were identified. The present study also supported the potential use of age at diagnosis as a risk factor for inherited RB.

Recently, NGS is considered a highly sensitive and efficient approach for the detection of $R B 1$ mutations due to increasing use of NGS in gene mutation analysis of RB; especially screening and identification of $R B 1$ mutations with NGS substantially benefits the prepotency, early diagnosis and treatment of retinoblastoma (20-25). Furthermore, the sensitivity rate of NGS in the present study was similar with that reported to previous studies. For example, Li et al (51) detected germline $R B 1$ mutations in $100 \%$ (19/19) of patients with bilateral RB, which was consistent with previously reported laboratory data. Additionally, Singh et al (52) demonstrated a detection rate of $100 \%$ (22/22) for bilateral cases, following validation of data using the TruSight Cancer Sequencing Panel (Illumina, Inc.), with a sensitivity rate of $98.2 \%$, specificity of $100 \%$, and reproducibility of $99.5 \%$. 
Table III. A total of 28 distinct $R B 1$ germline alterations, including 9 novel ones, were identified in 35 patients with retinoblastoma.

\begin{tabular}{|c|c|c|c|c|c|c|}
\hline$R B 1$ germline variants & $\begin{array}{c}\text { Molecular } \\
\text { consequences }\end{array}$ & $\begin{array}{l}\text { Amino acid } \\
\text { alterations }\end{array}$ & $\begin{array}{c}\text { Clinical } \\
\text { significance }\end{array}$ & $\begin{array}{l}\text { Existing } \\
\text { variations }\end{array}$ & $\begin{array}{l}\text { No. of } \\
\text { carriers }\end{array}$ & Subunits \\
\hline c.210_211insAG & Frameshift & p.(Ala74Glufs*4) & Pathogenic & Newly identified & 1 & N-terminus \\
\hline c. $306 \mathrm{~T}>\mathrm{A}$ & Nonsense & p.(Cys102Ter) & Pathogenic & Newly identified & 1 & N-terminus \\
\hline c.515_516insA & Frameshift & p.(Tyr173Ilefs*12) & Pathogenic & Newly identified & 1 & N-terminus \\
\hline $\begin{array}{l}\text { c.719-6_7192 } \\
\text { delTTACA }\end{array}$ & Splice & - & Pathogenic & Newly identified & 1 & N-terminus \\
\hline Ex12 Dup & $\begin{array}{l}\text { Large } \\
\text { rearrangement }\end{array}$ & - & Pathogenic & Newly identified & 1 & Pocket \\
\hline $\begin{array}{l}\text { c.1403_1404insA } \\
\text { c.1953_c.1960+1 }\end{array}$ & Frameshift & p.(Ser469Ilefs*6) & Pathogenic & Newly identified & 1 & Pocket \\
\hline delTAAAAAAGG & Splice & - & Pathogenic & Newly identified & 1 & Pocket \\
\hline c. $2106+1 \mathrm{G}>\mathrm{C}$ & Splice & - & Pathogenic & Newly identified & 1 & Pocket \\
\hline c. $2107-2 \mathrm{~A}>\mathrm{C}$ & Splice & - & Pathogenic & Newly identified & 1 & Pocket \\
\hline $\begin{array}{l}\text { c. } 83 \mathrm{C}>\mathrm{G} \text { (concurrent with } \\
\text { Ex12dup) }\end{array}$ & Missense & p.(Pro28Arg) & VUS & rs776175164 & 1 & N-terminus \\
\hline c. $1215+1 \mathrm{G}>\mathrm{A}$ & Splice & - & Pathogenic & rs587776783 & 1 & Pocket \\
\hline c.1953_1954insA & Frameshift & p.(V654Sfs*14) & Pathogenic & rs 1566234123 & 1 & Pocket \\
\hline c. $1981 \mathrm{C}>\mathrm{T}$ & Missense & p.(Arg661Trp) & Pathogenic & rs 137853294 & 1 & Pocket \\
\hline c. $2359 \mathrm{C}>\mathrm{T}$ & Nonsense & p.(Arg787Ter) & Pathogenic & rs 137853293 & 1 & Pocket \\
\hline c. $1735 \mathrm{C}>\mathrm{T}$ & Nonsense & p.(Arg579Ter) & Pathogenic & rs 121913305 & 3 & Pocket \\
\hline c. $1072 \mathrm{C}>\mathrm{T}$ & Nonsense & p.(Arg358Ter) & Pathogenic & rs 121913301 & 2 & N-terminus \\
\hline c. $.958 \mathrm{C}>\mathrm{T}$ & Nonsense & p.(Arg320Ter) & Pathogenic & rs 121913300 & 4 & N-terminus \\
\hline c. $2520+1 \_2520+4$ delGTGA & Splice & - & Pathogenic & rs 1131690858 & 1 & C-terminus \\
\hline Ex1_27 Del & $\begin{array}{l}\text { Large } \\
\text { rearrangement }\end{array}$ & - & Pathogenic & $(53,61-63)$ & 3 & Whole gene \\
\hline Ex18_23 Dup & $\begin{array}{l}\text { Large } \\
\text { rearrangement }\end{array}$ & - & Pathogenic & (64) & 1 & Pocket \\
\hline Ex24 Del & $\begin{array}{l}\text { Large } \\
\text { rearrangement }\end{array}$ & - & Pathogenic & (65) & 1 & C-terminus \\
\hline c. $1696-1 \mathrm{G}>\mathrm{A}$ & Splice & - & Pathogenic & COSV57329272 & 1 & Pocket \\
\hline c. $264+1 \mathrm{G}>\mathrm{C}$ & Splice & - & Pathogenic & COSV57317171 & 1 & N-terminus \\
\hline c. $1303 \mathrm{G}>\mathrm{T}$ & Nonsense & p.(Gly435Ter) & Pathogenic & COSV57313162 & 1 & Pocket \\
\hline c. $607+1 \mathrm{G}>\mathrm{A}$ & Splice & - & Pathogenic & COSV57310480 & 1 & N-terminus \\
\hline c. $607+1 \mathrm{G}>\mathrm{T}$ & Splice & - & Pathogenic & COSV57310480 & 1 & N-terminus \\
\hline c. $380 \mathrm{G}>\mathrm{A}$ & Missense & p.(Ser127Asn) & Pathogenic & COSV57302882 & 1 & N-terminus \\
\hline c. $1128-1 \mathrm{G}>\mathrm{A}$ & Splice & - & Pathogenic & COSV57294096 & 1 & Pocket \\
\hline
\end{tabular}

RB1, RB transcriptional corepressor 1; Ex, exon; Ins, insertion; Del, deletion; Dup, duplication.

Furthermore, Grotta et al (53) showed a determination rate of $96 \%$ (28/29) for patients with bilateral RB when combining NGS and aCGH. Herein, the detected $R B 1$ variants had common characteristics with $R B 1$ alterations commonly observed in patients with RB. For instance, mutations were not identified in the exons 26 and 27 of the $R B 1$ gene and were scattered along the $R B 1$ gene. Another study revealed that null mutations, including large rearrangements, nonsense, splicing and frameshift mutations were detected at high frequency, whereas missense mutations exhibited a low frequency (6). Overall, the aforementioned data indicated that NGS could be considered as an accurate method for detecting germline $R B 1$ mutations.

The detection rate of germline $R B 1$ mutations in Vietnamese patients with RB using SS-MLPA has been previously reported.
Therefore, Kiet et al (26) and Nguyen et al (27) detected germline $R B 1$ mutations in $84(21 / 25)$ and $82 \%(9 / 11)$ of patients with bilateral RB, respectively. However, the detection rates were lower compared with those reported in other Asian studies applying the same approach. For example, Tomar et al (18) and $\mathrm{He}$ et al (54) revealed a detection rate of 94 (17/18) and 92\% (36/39) in Singaporean and Chinese patients with bilateral RB, respectively. In addition, Rojanaporn et al (55) and Mohd Khalid et al (56) also reported a detection rate of germline RB1 mutations of $92(25 / 27)$ and $100 \%(7 / 7)$ in Thai and Malaysian patients with bilateral RB, respectively.

In the present study nine novel $R B 1$ mutations were identified in the cyclin fold-contained subunits of the pRB. The allele frequencies of these null mutations were $<0.0001 \%$ in the public 
Table IV. Distribution of $R B 1$ and non- $R B 1$ carriers diagnosed at 0-36 vs. >36 months.

A, All patients with RB

\begin{tabular}{lccr}
\hline Characteristic & $R B 1$ carriers, $\mathrm{n}=35$ & Non- $R B 1$ carriers, $\mathrm{n}=27$ & Total \\
\hline Age, $\mathrm{n}(\%)$ & & & $0.036^{\mathrm{a}}$ \\
$0-36$ months & $31(63.3)$ & $18(36.7)$ & $49(100.0)$ \\
$>36$ months & $4(36.0)$ & $9(64.0)$ & $13(100.0)$ \\
OR, $0-36$ vs. $>36$ months & $3.88(95 \% \mathrm{CI}, 1.04-14.40)$ & $0.26(95 \% \mathrm{CI}, 0.07-0.96)$ & 0.040 \\
\hline
\end{tabular}

B, Patients with unilateral RB only

\begin{tabular}{lcrr}
\hline Characteristic & $R B 1$ carriers, $\mathrm{n}=10$ & Non- $R B 1$ carriers, $\mathrm{n}=27$ & Total \\
\hline Age, $\mathrm{n}(\%)$ & & & 0.62 \\
$0-36$ months & $8(30.8)$ & $18(69.2)$ & $0.043^{\mathrm{a}}$ \\
$>36$ months & $2(18.0)$ & $9(82.0)$ & $11(100.0)$ \\
OR, $0-36$ vs. $>36$ months & $2(95 \%$ CI, $0.35-11.40)$ & $0.5(95 \% \mathrm{CI}, 0.09-2.86)$ & 0.043 \\
\hline
\end{tabular}

${ }^{a}$ Calculated using Fisher's exact test. OR, odds ratio; RB, retinoblastoma; RB1, RB transcriptional corepressor 1.

databases, and had notbeen recorded in the genetic polymorphism database consisting of 400 healthy Vietnamese individuals (47). The low frequency of these variations in the general population indicated that these mutations could be eliminated during evolution due to their disadvantages. Additionally, the CADD tool predicted that all these alterations, except one (EX12 DUP), were among the top $1 \%$ of deleterious variants in the human genome. CADD integrates multiple annotations, including genomic features, gene-annotation models, evolutionary and epigenetic features, and gene functional predictions to generate a single, quantitative scoring system. These scores are then used to rank the deleterious effect of a given variant $(48,57)$. For example, a CADD score of 20 or 30 suggests that a variant is among the top $1 \%$ or $0.1 \%$ of deleterious variants in the human genome, respectively. Consequently, VarSome (44), a tool for implementing the ACMG standards, is used to classify variants as pathogenic ones when they meet very strong evidence of pathogenicity (PSV1). The PSV1 criterion assumes that certain null mutations, such as nonsense, frameshift, splice sites of $+/-1$ or 2 , initiation codon and exon deletions, can lead to a complete absence of the gene product due to impaired transcription or nonsense-mediated decay of an altered transcript (35). The predicted null variants in a gene, whose loss of function is the known mechanism underlying the development of a particular disease, such as $R B 1$ for $\mathrm{RB}$, can be considered as the common cause of the disease. Devarajan et al (58) also applied the stringent criteria for defining pathogenic variants and reported no false-positive results during the detection of constitutional $R B 1$ variants.

The two-hit hypothesis suggests that in patients with hereditary RB the tumors are formed at younger ages compared with sporadic cases (28). In 1998, Zajaczek et al (31) reported four patients with unilateral RB, who were diagnosed with germline $R B 1$ mutations at the age of $<19$ months. This initial evidence supported the hypothesis that age at diagnosis could differentiate the hereditary from the sporadic form of unilateral
RB. However, further studies with larger sample sizes did not reveal any association between age at diagnosis and germline $R B 1$ status in patients with unilateral RB (32-34). In addition, Tomar et al (18) showed that Singaporean individuals diagnosed with RB at 0-36 months of age had a 53\% risk of being $R B 1$ carriers, whereas those $>36$ months had $8 \%$ risk of suffering from hereditary $\mathrm{RB}$. The results of the present study were consistent with those of the previous one, suggesting that children diagnosed with RB at 0-36 months of age were more likely to be $R B 1$ carriers compared with those aged $>36$ months. Nevertheless, similar analyses in patients with unilateral RB did not reveal significant difference in the risk of hereditary RB.

The present study revealed three clinical implications. Firstly, the results supported that the NGS method implemented at the Vinmec Hi-Tech Center was highly reliable in detecting germline $R B 1$ variants. Secondly, this study could provide novel insights into the mechanisms underlying $R B 1$ inactivation. Finally, the current findings suggested that age at diagnosis could be considered as a risk factor for hereditary RB.

Nevertheless, there are some limitations in the present retrospective study, including the small sample sizes and delay in diagnosis. Therefore, the results could not be generalizable to all patients with RB. The number of patients with bilateral RB, who underwent $R B 1$ testing in each of the aforementioned studies was $<30$. Furthermore, the small sample size and different detection strategies used to identify germline $R B 1$ mutations could result in variations in the detection rate between NGS and SS-MPLA. However, previous studies revealed that NGS-based methods could detect germline $R B 1$ mutations in patients with $\mathrm{RB}$, whose constitutional $R B 1$ mutations could not be detected by either Sanger sequencing nor MPLA $(59,60)$. In addition, the lack of statistical significance in the relative risk of patients with unilateral RB diagnosed at the age of 0-36 and those 
>36 months being carriers of RB1 could be also due to the small number of unilateral cases.

In conclusion, the results of the present study indicated that NGS could be considered a reliable method for screening for constitutional $R B 1$ mutations, and age at diagnosis could be used to assess the risk of hereditary RB. Furthermore, the newly identified $R B 1$ mutations could provide useful information for an in-depth understanding of the mechanisms underlying $R B 1$ inactivation, and for the development of rapid assays for detecting $R B 1$ mutations. Altogether, the current study suggested that NGS could be used for detecting germline $R B 1$ mutations in routine clinical practice.

\section{Acknowledgements}

The authors would like to thank Professor Paolo Boffetta (Icahn School of Medicine, Mount Sinai School of Medicine, Tisch Cancer Institute, New York City, NY, USA) for the critical review of this manuscript.

\section{Funding}

No funding was received.

\section{Availability of data and materials}

The FASTQ data that support the findings of this study are available from the Beijing Genomics Institute (BGI) laboratory (Hong Kong) but restrictions apply to the availability of these data, which were used under license for the current study, and so are not publicly available. Data are however available from the authors upon reasonable request and with permission of the BGI laboratory.

\section{Authors' contributions}

CQH and HQD conceived and designed the study. BDN, LTP, DTN, CTMP and TLD recruited and referred patients to the study and helped with their follow-up. HQD, $\mathrm{CQH}$, NTN, SAHN, CN, BDN, LTP, DTN, CTMP, TLD and MHT performed the experiments. HQD, CQH, NTN, SAHN, CN and MHT analyzed and interpreted the results. $\mathrm{CQH}$ and HQD wrote the manuscript. HQD and CQH are responsible for confirming the authenticity of the raw data. All authors read and approved the final version of the manuscript.

\section{Ethics approval and consent to participate}

Signed informed consents were obtained from parents/caregivers of all subjects, and the study was approved by the Vinmec's Institutional Review Board (Vinmec Healthcare System, Hanoi, Vietnam).

\section{Patient consent for publication}

Not applicable.

\section{Competing interests}

The authors declare that they have no competing interests.

\section{References}

1. Canty CA: Retinoblastoma: An overview for advanced practice nurses. J Am Acad Nurse Pract 21: 149-155, 2009.

2. Aerts I, Lumbroso-Le Rouic L, Gauthier-Villars M, Brisse H, Doz F and Desjardis L: Retinoblastoma. Orphanet J Rare Dis 1: 31, 2006.

3. Kim JY and Park Y: Treatment of retinoblastoma: The role of external beam radiotherapy. Yonsei Med J 56: 1478-1491, 2015.

4. Abramson DH: Retinoblastoma: Saving life with vision. Annu Rev Med 65: 171-184, 2014.

5. Soliman SE, Racher H, Zhang C, MacDonald H and Gallie BL: Genetics and molecular diagnostics in retinoblastoma-an update. Asia Pac J Ophthalmol (Phila) 6: 197-207, 2017.

6. Valverde JR, Alonso J, Palacios I and Pestaňa A: RB1 gene mutation up-date, a meta-analysis based on 932 reported mutations available in a searchable database. BMC Genet 6: 53, 2005.

7. Draper GJ, Sanders BM, Brownbill PA and Hawkins MH: Patterns of risk of hereditary retinoblastoma and applications to genetic counselling. Br J Cancer 66: 211-219, 1992.

8. Rushlow D, Piovesan B, Zhang K, Prigoda-Lee NL, Marchong MN, Clark RD and Gallie BL: Detection of mosaic RB1 mutations in families with retinoblastoma. Hum Mutat 30: 842-851, 2009.

9. Ali A, Kletke S, Gallie B and Lam WC: Retinoblastoma for pediatric ophthalmologists. Asia Pac J Ophthalmol (Phila) 7: 160-168, 2018.

10. Canadian Retinoblastoma Society: National retinoblastoma strategy Canadian guidelines for care. Can J Ophthalmol 44 (Suppl 2): S9-S47, 2009.

11. Dhar SU, Chintagumpala M, Noll C, Chévez-Barrios P, Paysse EA and Plon SE: Outcomes of integrating genetics in management of patients with retinoblastoma. Arch Ophthalmol 129: 1428-1434, 2011.

12. Joseph B, Shanmugam MP, Srinivasan MK and Kumaramanickavel G: Retinoblastoma: Genetic testing versus conventional clinical screening in India. Mol Diagn 8: 237-243, 2004.

13. Berry JL, Kim JW, Damato BE and Singh AD (eds): Clinical Ophthalmic Oncology: Retinoblastoma. Springer, p303, 2019.

14. Ali MJ, Parsam VL, Honavar SG, Kannabiran C, Vemuganti GK and Reddy VA: RB1 gene mutations in retinoblastoma and its clinical correlation. Saudi J Ophthalmol 24: 119-123, 2010.

15. Lohmann DR: RB1 gene mutations in retinoblastoma. Hum Mutat 14: 283-288, 1999.

16. Ellison G, Donald E, McWalter G, Knight L, Fletcher L, Sherwood J, Cantarini M, Orr M and Speake G: A comparison of ARMS and DNA sequencing for mutation analysis in clinical biopsy samples. J Exp Clin Cancer Res 29: 132, 2010.

17. Richter S, Vandezande K, Chen N, Zhang K, Sutherland J, Anderson J, Han L, Panton R, Branco P and Gallie B: Sensitive and efficient detection of RB1 gene mutations enhances care for families with retinoblastoma. Am J Hum Genet 72: 253-269, 2003.

18. Tomar S, Sethi R, Sundar G, Quah TC, Quah BL and Lai PS: Mutation spectrum of RB1 mutations in retinoblastoma cases from Singapore with implications for genetic management and counselling. PLoS One 6: e0178776, 2017.

19. Thirumalairaj K, Abraham A, Devarajan B, Gaikwad N, Kim U, Muthukkaruppan V and Vanniarajan A: A stepwise strategy for rapid and cost-effective RB1 screening in Indian retinoblastoma patients. J Hum Genet 60: 547-552, 2015.

20. Mehyar M, Mosallam M, Tbakhi A, Saab A, Sultan I, Deebajah R, Jaradat I, AlJabari R, Mohammad M, AlNawaiseh I, et al: Impact of RB1 gene mutation type in retinoblastoma patients on clinical presentation and management outcome. Hematol Oncol Stem Cell Ther 13: 152-159, 2020.

21. Zhang Z, Xiao YS, Shen R, Jiang HC, Tan L, Li RQ, Yang XH, $\mathrm{Gu} \mathrm{HY}, \mathrm{He}$ WJ and Ma J: Next generation sequencing of RB1 gene for the molecular diagnosis of ethnic minority with retinoblastoma in Yunnan. BMC Med Genet 21: 230, 2020.

22. Xu L, Shen L, Polski A, Prabakar RK, Shah R, Jubran R, Kim JW, Biegel J, Kuhn P, Cobrinik D, et al: Simultaneous identification of clinically relevant RB1 mutations and copy number alterations in aqueous humor of retinoblastoma eyes. Ophthalmic Genet 41: 526-532, 2020.

23. Chai P, Luo Y, Yu J, Li Y, Yang J, Zhuang A, Fan J, Han M and Jia R: Clinical characteristics and germline mutation spectrum of RB1 in Chinese patients with retinoblastoma: A dual-center study of 145 patients. Exp Eye Res 205: 108456, 2021. 
24. Zou Y, Li J, Hua P, Liang T, Ji X and Zhao P: Spectrum of germline mutations in RB1 in Chinese patients with retinoblastoma: Application of targeted next-generation sequencing. Mol Vis 27: $1-16,2021$.

25. Francis JH, Richards AL, Mandelker DL, Berger MF, Walsh MF, Dunkel IJ, Donoghue MTA and Abramson DH: Molecular changes in retinoblastoma beyond RB1: Findings from next-generation sequencing. Cancers (Basel) 13: 149, 2021.

26. Kiet NC, Khuong LT, Minh DD; Nguyen The Vinh, Quan NHM, Xinh PT, Trang NNC, Luan NT, Khai NM and Vu HA: Spectrum of mutations in the RB1 gene in Vietnamese patients with retinoblastoma. Mol Vis 25: 215-221, 2019.

27. Nguyen HH, Nguyen HTT, Vu NP, Le QT, Pham CM, Huyen TT, Manh H, Pham HLB, Nguyen TD, Le HTT and Van Nong $\mathrm{H}$ Mutational screening of germline RB1 gene in Vietnamese patients with retinoblastoma reveals three novel mutations. Mol Vis 24: 231-238, 2018

28. Knudson AG Jr: Mutation and cancer: Statistical study of retinoblastoma. Proc Natl Acad Sci USA 68: 820-823, 1971

29. Dimaras H, Kimani K, Dimba EA, Gronsdahl P, White A, Chan HS and Ballie BL: Retinoblastoma. Lancet 379: 1436-1446, 2012.

30. Lohmann DL and Gallie BL: Retinoblastoma: Revisiting the model prototype of inherited cancer. Am J Med Genet C Semin Med Genet 129C: 23-28, 2004.

31. Zajaczek S, Jakubowska A, Kurzawski G, Krzystolik Z and Lubiński J: Age at diagnosis to discriminate those patients for whom constitutional DNA sequencing is appropriate in sporadic unilateral retinoblastoma. Eur J Cancer 34: 1919-1921, 1998.

32. Schüler A, Weber S, Neuhäuser M, Jurklies C, Lehnert T, Heimann H, Rudolph G, Jöckel KH, Bornfeld N and Lohmann DR: Age at diagnosis of isolated unilateral retinoblastoma does not distinguish patients with and without a constitutional RB1 gene mutation but is influenced by a parent-of-origin effect. Eur J Cancer 41: 735-740, 2005

33. Lohmann DR, Gerick M, Brandt B, Oelschläger U, Lorenz B, Passarge E and Horsthemke B: Constitutional RB1-gene mutations in patients with isolated unilateral retinoblastoma. Am J Hum Genet 61: 282-294, 1997.

34. Berry JL, Lewis L, Zolfaghari E, Green S, Le BHA, Lee TC, Murphree AL, Kim JW and Jubran R: Lack of correlation between age at diagnosis and RB1 mutations for unilateral retinoblastoma: The importance of genetic testing. Ophthalmic Genet 39: 407-409, 2018

35. Richards S, Aziz N, Bale S, Bick D, Das S, Gastier-Foster J, Brody WW, Hegde M, Lyon E, Spector E, et al: Standards and guidelines for the interpretation of sequence variants: A joint consensus recommendation of the American college of medical genetics and genomics and the association for molecular pathology. Genet Med 17: 405-424, 2015.

36. den Dunnen JT, Dalgleish R, MaglottDR,Hart RK, GreenblattMS, McGowan-Jordan J, Roux AF, Smith T, Antonarakis SE and Taschner PE: HGVS recommendations for the description of sequence variants: 2016 update. Hum Mutat 37: 564-569, 2016.

37. Landrum MJ, Lee JM, Riley GR, Jang W, Rubinstein WS Church DM and Maglott DR: ClinVar: Public archive of relationships among sequence variation and human phenotype. Nucleic Acids Res 42: D980-D985, 2014.

38. Béroud C, Collod-Béroud G, Boileau C, Soussi T and Junien C: UMD (Universal mutation database): A generic software to build and analyze locus-specific databases. Hum Mutat 15: 86-94, 2000

39. Fokkema IF, Taschner PE, Schaafsma GC, Celli J, Laros JF and den Dunnen JT: LOVD v.2.0: The next generation in gene variant databases. Hum Mutat 32: 557-563, 2011.

40. ARUP Scientific Resource for Research and Education. Available from: https://arup.utah.edu/.

41. Vaser R, Adusumalli S, Leng SN, Sikic M and Ng PC: SIFT missense predictions for genomes. Nat Protoc 11: 1-9, 2016.

42. Adzhubei IA, Schmidt S, Peshkin L, Ramensky VE, Gerasimova A, Bork P, Kondrashov AS and Sunyaev SR: A method and server for predicting damaging missense mutations. Nat Methods 7: 248-249, 2010

43. Schwarz JM, Cooper DN, Schuelke M and Seelow D MutationTaster2: Mutation prediction for the deep-sequencing age. Nat Methods 11: 361-362, 2014.

44. Kopanos C, Tsiolkas V, Kouris A, Chapple CE, Aguilera MA, Meyer R and Massouras A: VarSome: The human genomic variant search engine. Bioinformatics 35: 1978-1980, 2019.

45. Li Q and Wang $\mathrm{K}$ : InterVar: Clinical interpretation of genetic variants by the 2015 ACMG-AMP guidelines. Am J Hum Genet 100: 267-280, 2017.
46. McLaren W, Gil L, Hunt SE, Riat HS, Ritchie GS, Thormann A, Flicek $P$ and Cunningham F: The ensembl variant effect predictor. Genome Biol 17: 122, 2016.

47. Le VS, Tran KT, Bui HTP, Le HTT, Nguyen CD, Do DH, Ly HTT, Pham LTD, Dao LTM and Nguyen LT: A Vietnamese human genetic variation database. Hum Mutat 40: 1664-1675, 2019.

48. Rentzsch P, Witten D, Cooper GM, Shendure J and Kircher M: CADD: Predicting the deleteriousness of variants throughout the human genome. Nucleic Acids Res 47: D886-D894, 2019.

49. Social Science Statistics. Available from: https://www.socscistatistics.com/.

50. Schoonjans F, Zalata A, Depuydt CE and Comhaire FH: MedCalc: A new computer program for medical statistics. Comput Methods Programs Biomed 48: 257-262, 1995.

51. Li WL, Buckley J, Sanchez-Lara PA, Maglinte DT, Viduetsky L, Tatarinova TV, Aparicio JG, Kim JW, Au M, Ostrow D, et al: A rapid and sensitive next-generation sequencing method to detect RB1 mutations improves care for retinoblastoma patients and their families. J Mol Diagn 18: 480-493, 2016.

52. Singh J, Mishra A, Pandian AJ, Mallipatna AC, Khetan V, Sripriya S, Kapoor S, Agarwal S, Sankaran S, Katragadda S, et al: Next-generation sequencing-based method shows increased mutation detection sensitivity in an Indian retinoblastoma cohort. Mol Vis 22: 1036-1047, 2016.

53. Grotta S, D'Elia G, Scavelli R, Genovese S, Surace C, Sirleto P, Cozza R, Romanzo A, De lois MA, Valente P, et al: Advantages of a next generation sequencing targeted approach for the molecular diagnosis of retinoblastoma. BMC Cancer 15: 841, 2015.

54. He MY, An Y, Gao YJ, Qian XW, Li G and Qian J: Screening of RB1 gene mutations in Chinese patients with retinoblastoma and preliminary exploration of genotype-phenotype correlations. Mol Vis 20: 545-552, 2014

55. Rojanaporn D, Boontawon $\mathrm{T}$, Chareonsirisuthigul $\mathrm{T}$, Thanapanpanich O, Attaseth T, Saengwimol D, Anurathapan U, Sujirakul T, Kaewkhaw R and Hongeng S: Spectrum of germline RB1 mutations and clinical manifestations in retinoblastoma patients from Thailand. Mol Vis 24: 778-788, 2018

56. Mohd Khalid MK, Yakob Y, Md Yasin R, Teik K, Siew CG, Rahmat J, Ramasamy S and Alagaratnam J: Spectrum of germ-line RB1 gene mutations in Malaysian patients with retinoblastoma. Mol Vis 21: 1185-1190, 2015.

57. Kircher M, Witten DM, Jain P, O'Roak BJ, Cooper GM and Shendure J: A general framework for estimating the relative pathogenicity of human genetic variants. Nat Genet 46: 310-315, 2014.

58. Devarajan B, Prakash L, Kannan TR, Abraham AA, Kim U, Muthukkaruppan V and Vanniarajan A: Targeted next generation sequencing of RB1 gene for the molecular diagnosis of Retinoblastoma. BMC Cancer 15: 320, 2015.

59. Chen Z, Moran K, Richards-Yutz J, Toorens E, Gerhart D, Ganguly T, Shields CL and Ganguly A: Enhanced sensitivity for detection of low-level germline mosaic RB1 mutations in sporadic retinoblastoma cases using deep semiconductor sequencing. Hum Mutat 35: 384-391, 2014.

60. Rodríguez-Martín C, Robledo C, Gómez-Mariano G, Monzón S, Sastre A, Abelairas J, Sábado C, Martín-Begué N, Ferreres JC, Fernández-Teijeiro A, et al: Frequency of low-level and high-level mosaicism in sporadic retinoblastoma: Genotype-phenotype relationships. J Hum Genet 65: 165-174, 2020.

61. Parsam VL, Kannabiran C, Honavar S, Vemuganti GK and Ali MJ: A comprehensive, sensitive and economical approach for the detection of mutations in the RB1 gene in retinoblastoma. J Genet 88: 517-527, 2009

62. Parma D, Ferrer M, Luce L, Giliberto F and Szijan I: RB1 gene mutations in Argentine retinoblastoma patients. Implications for genetic counseling. PLoS One 12: e0189736, 2017.

63. Lan X, Xu W, Tang X, Ye H, Song X, Lin L, Ren X, Yu G, Zhang $\mathrm{H}$ and Wu S: Spectrum of RB1 germline mutations and clinical features in unrelated Chinese patients with retinoblastoma. Front Genet 11: 142, 2020.

64. Berge EO, Knappskog S, Lillehaug JR and Lønning PE: Alterations of the retinoblastoma gene in metastatic breast cancer. Clin Exp Metastasis 28: 319-326, 2011.

65. Kato MV, Ishizaki K, Toguchida J, Kaneko A, Takayama J, Tanooka H, Kato T, Shimuzu T and Sasaki MS: Mutations in the retinoblastoma gene and their expression in somatic and tumor cells of patients with hereditary retinoblastoma. Hum Mutat 3: 44-51, 1994.

66. Fabian ID, Reddy A and Sagoo MS: Classification and staging of retinoblastoma. Community Eye Health 31: 11-13, 2018. 